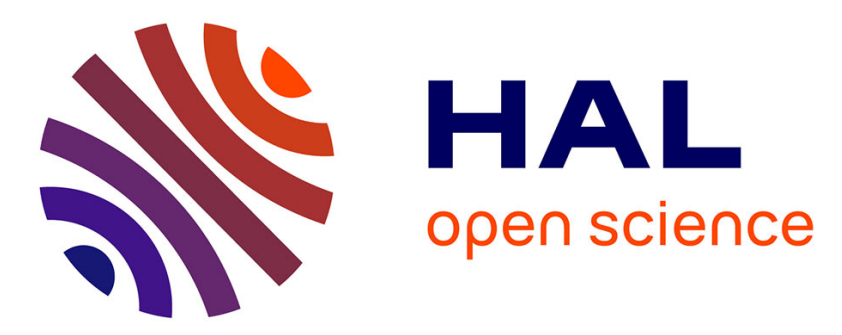

\title{
Propriétés optiques et aberrations d'un canon à émission de champ pour la microscopie électronique à 75 et 100 kv
}

\author{
M. Troyon, N. Bonnet, F. Payen
}

\section{- To cite this version:}

M. Troyon, N. Bonnet, F. Payen. Propriétés optiques et aberrations d'un canon à émission de champ pour la microscopie électronique à 75 et $100 \mathrm{kv}$. Revue de Physique Appliquée, 1972, 7 (1), pp.3-9. 10.1051/rphysap:01972007010300 . jpa-00243594

\section{HAL Id: jpa-00243594 https://hal.science/jpa-00243594}

Submitted on 1 Jan 1972

HAL is a multi-disciplinary open access archive for the deposit and dissemination of scientific research documents, whether they are published or not. The documents may come from teaching and research institutions in France or abroad, or from public or private research centers.
L'archive ouverte pluridisciplinaire HAL, est destinée au dépôt et à la diffusion de documents scientifiques de niveau recherche, publiés ou non, émanant des établissements d'enseignement et de recherche français ou étrangers, des laboratoires publics ou privés. 


\title{
PROPRIÉTÉS OPTIQUES \\ ET ABERRATIONS D'UN CANON A ÉMISSION DE CHAMP POUR LA MICROSCOPIE ÉLECTRONIQUE A 75 ET $100 \mathrm{kV}$
}

\author{
M. TROYON, N. BONNET et F. PAYEN \\ Laboratoire d'Electricité, Faculté des Sciences, BP 347, Moulin de la Housse, 51-Reims
}

(Reçu le 21 juillet 1971)

\begin{abstract}
Résumé. - Les propriétés optiques, les aberrations sphérique et chromatique de différents canons à électrons à émission de champ pour la microscopie électronique à $75 \mathrm{kV}$ et $100 \mathrm{kV}$ sont étudiées expérimentalement par une méthode analogique. Cette étude permet de choisir le canon qui donne les aberrations minimums, donc la brillance $\left({ }^{*}\right)$ du faisceau électronique maximum. Celle-ci a une valeur de l'ordre de $1 \times 10^{9} \mathrm{~A} / \mathrm{cm}^{2}$ stérad. pour un champ électrique appliqué sur la pointe de la cathode égal à $4,5 \times 10^{7} \mathrm{~V} / \mathrm{cm}$, la tension d'accélération des électrons étant $100 \mathrm{kV}$.

Abstract. - Optical properties, spherical and chromatic aberrations of different field emission electron gun for 75 and $100 \mathrm{kV}$ electron microscopy are experimentaly investigated using an analogical method. This investigation allows to choose the gun which gives minimum aberrations and consequently the maximum electron beam brightness. This brightness is approximatively $10^{9} \mathrm{~A} / \mathrm{cm}^{2}$ sterad. when the field at the cathode surface is $4.5 \times 10^{7} \mathrm{~V} / \mathrm{cm}$ and the accelerating voltage $100 \mathrm{kV}$.
\end{abstract}

1. Introduction. - Des études théoriques (Everhart [1], Worster [2], Butler [3]) et expérimentales (Crewe [4]) de canons à émission de champ pour l'utilisation dans un microscope électronique ont déjà été réalisées.

Everhart a fait les hypothèses suivantes : entre la cathode et la première anode il suppose que la répartition du potentiel électrique est sphérique, et entre les deux anodes qui constituent l'étage accélérateur qu'elle est de révolution autour de l'axe du canon, la méridienne étant une hyperbole. Dans cette étude Everhart ne tient pas compte du cône qui soutient l'extrémité de la pointe et de son effet sur le champ dans le voisinage de celle-ci. Dans une étude plus récente [5], ce même auteur a repris les hypothèses que Dyke [6] avait faites sur les formes des pointes pour tenir compte de cet effet.

Worster quant à lui, a voulu aussi tenir compte de cet effet en supposant que l'extrémité de la cathode et la première anode sont des paraboloïdes de révolution de même foyer. En fait les résultats d'Everhart et de Worster sont assez voisins et tendent à montrer que les coefficients d'aberration dans le voisinage immédiat de la pointe sont négligeables devant les aberrations du canon pris dans son ensemble.

Crewe [7], partant de l'hypothèse que les aberrations sont dues principalement à l'effet de lentille créé

(*) Certains auteurs préfèrent dire luminance. Nous emploierons le mot brillance par analogie avec le mot anglo-saxon. par les diaphragmes des anodes $(* *)$ a cherché une forme d'anode telle que cet effet disparaisse. Butler [3] a fait l'étude théorique à l'ordinateur et Crewe l'étude expérimentale mais seulement à des tensions d'accélérations faibles de l'ordre de $30 \mathrm{kV}$.

Dans le but d'équiper un microscope électronique par transmission avec un canon à émission de champ nous avons recherché pour différentes formes d'anodes semblables au modèle de Butler, les conditions optimums d'utilisation pour des potentiels accélérateurs de $75 \mathrm{kV}$ et $100 \mathrm{kV}$ [8]. Nos premiers résultats concernant la réalisation expérimentale du canon à émission de champ ainsi que des tests de stabilité du courant d'émission ont été présentés dans une thèse de $3^{\mathrm{e}}$ cycle [9]. Nous reprenons ici plus particulièrement les résultats concernant l'étage accélérateur.

2. Description de la méthode employée. - Nous avons divisé le canon en trois régions (voir Fig. 1a) pour lesquelles la répartition du potentiel est très différente.

La région $I$ s'étend sur une distance très courte $z=10 R$ ( $R$ étant le rayon de la pointe). Dans cette région très proche de l'extrémité de la pointe le gradient

\footnotetext{
(**) L'effet de lentille se produit lorsque le module du champ électrique de part et d'autre du diaphragme est différent de zéro. Cet effet, et donc l'aberration qu'il crée, est d'autant plus fort que le gradient du potentiel change brusquement de chaque côté du diaphragme.
} 
du potentiel est très grand et les électrons sont soumis aux effets d'un champ divergent.

Dans la région II qui s'étend jusqu'à la première anode, au contraire, le gradient du potentiel est très faible et les électrons sont soumis aux effets d'un champ très légèrement convergent.

C'est dans la région III interanodique que l'on a l'effet de lentille proprement dit, et l'action de convergence $y$ est forte.

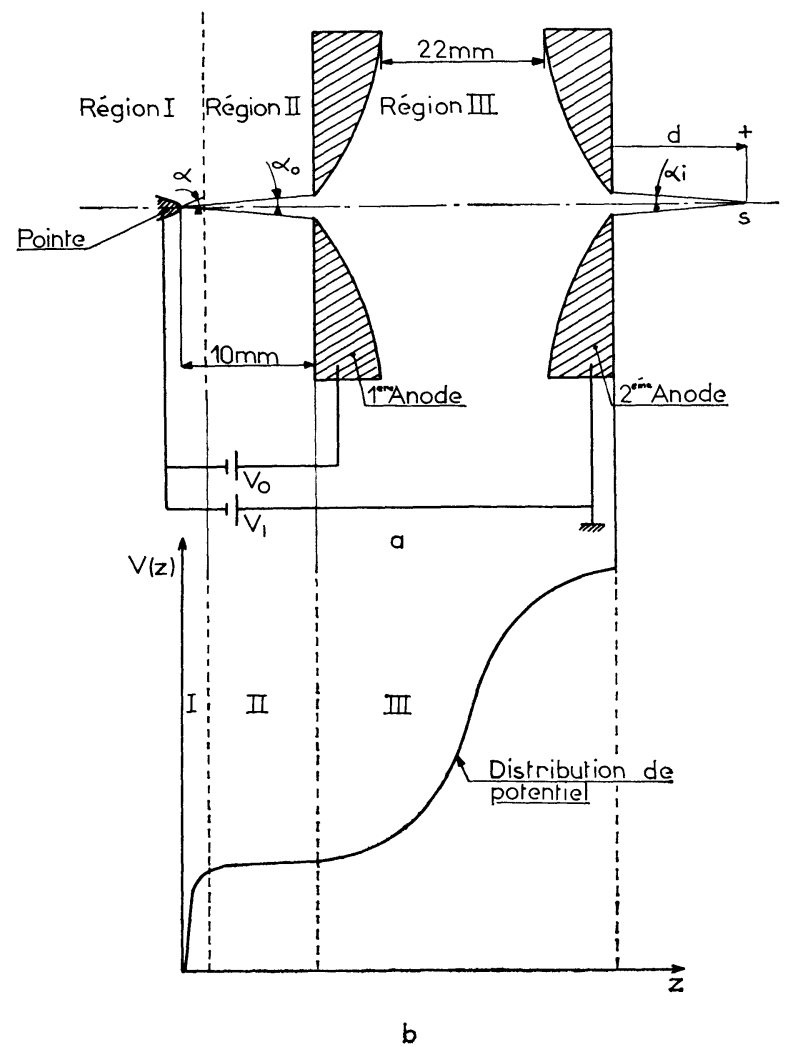

Fig. 1. - a) Diagramme schématique du canon à émission de champ. La tension $V_{0}$ entre la cathode et la première anode contrôle le courant émis par la pointe du filament de tungstène. La tension $V_{\boldsymbol{i}}$ entre la cathode et la seconde anode détermine l'énergie finale des électrons. La région I n'est pas à l'échelle elle s'étend sur une distance $10 R$ ( $R$ est le rayon de la pointe). b) Représentation de la distribution de potentiel sur l'axe du canon.

Dans la région I nous avons supposé comme Everhart [1] que la répartition du potentiel était sphérique.

Ensuite par une intégration numérique à l'ordinateur de l'équation des trajectoires paraxiales nous avons calculé les grandeurs électrooptiques et les coefficients d'aberration du canon. Cette intégration numérique n'étant valable que dans l'approximation de gauss nous l'avons faite commencer à une distance $z=10 R$ de l'extrémité de la cathode, distance pour laquelle les trajectoires sont paraxiales. En effet Everhart montre que, pour $z=10 R, 99 \%$ de l'énergie cinétique transverse initiale est transformée en énergie cinétique radiale et que les trajectoires électroniques qui partent au voisinage de l'axe font un angle maximum avec celui-ci d'environ $10^{-2}$ radian. Nous négligeons donc dans notre intégration numérique l'influence de cette région I proche de la cathode : cette région s'étend sur une distance très courte et l'aberration qu'elle crée est négligeable devant l'aberration totale du canon.

Pour l'étude de la région II, les dimensions de la pointe étant petites par rapport au pas de notre réseau de résistances, nous avons employé une cuve électrolytique. Nous avons symbolisé la cathode par une épingle dont on a mesuré le rayon de la pointe au microscope optique et la première anode par un disque plan. Les mesures, faites à la cuve électrolytique, sont de précision inférieure à celles faites au réseau de résistances, mais nous avons amélioré cette précision en faisant un lissage de la répartition de potentiel expérimentale obtenue dans la région II. La précision de nos résultats, quoique difficilement chiffrable, semble très satisfaisante car nous n'avons relevé aucune valeur aberrante des coefficients d'aberration sphérique et chromatique. En effet les valeurs expérimentales de ces coefficients d'aberration s'inscrivent parfaitement sur les courbes (Fig. 7).

Nous avons également voulu déterminer l'influence de la répartition du potentiel entre la cathode et la première anode sur le résultat global. Pour cela, nous avons remplacé la répartition de potentiel expérimentale correspondant à la région II par la répartition de potentiel théorique de Becker [10] qui correspond à une cathode et une première anode ayant les formes de paraboloïdes de révolution de même foyer. Les résultats obtenus pour la forme d'anode $\mathrm{D}$ à $100 \mathrm{kV}$ (voir tableau III) montrent une amélioration du coefficient d'aberration sphérique, de $20 \%$ et du coefficient d'aberration chromatique de $10 \%$ par rapport à nos résultats expérimentaux. On peut donc constater que la répartition $\mathrm{du}$ potentiel entre cathode et première anode, donc la forme de celles-ci a une influence sur les performances du canon. On pourrait alors penser à rechercher la géométrie optimum de la première anode, en regard de la cathode. Cette étude ne se justifie pas vis-à-vis du gain qu'elle peut apporter pour deux raisons :

- la connaissance exacte de la géométrie de la cathode est très difficile car suivant les conditions de formation de la pointe (11), la forme de celle-ci varie ;

- l'usinage de la première anode est très difficile, surtout si l'on veut placer un diaphragme à cet endroit.

3. Considérations sur la brillance et la largeur de la source électronique. - La brillance est directement liée à la largeur apparente de la source électronique comme la formule (12) ci-dessous le montre. Pour déterminer cette largeur apparente de source les aberrations du canon doivent être évaluées. Dans le plan image de gauss le rayon $r_{\mathrm{g}}$ de l'image de la pointe de la cathode est :

$$
r_{\mathbf{g}}=m r
$$


$m$ étant le grandissement linéaire du canon et $r$ le rayon apparent de la pointe, valant approximativement (1)

$$
r=R\left(\frac{V_{\mathrm{t}}}{R E_{R}}\right)^{1 / 2}
$$

où $R$ est le rayon de la pointe, $E_{R}$ le champ électrique appliqué à la pointe et $e V_{\mathrm{t}}$ l'énergie transverse moyenne des électrons quittant la cathode $(\sim 0,1 \mathrm{eV}$ pour $\left.E_{R}=4 \times 10^{7} \mathrm{~V} / \mathrm{cm}\right)$.

Les effets des aberrations dans le plan image de gauss sont les suivants :

- le rayon du disque d'aberration sphérique est

$$
r_{\mathrm{s}}=m C_{\mathrm{s}} \alpha^{3}
$$

$C_{\mathrm{s}}$ étant le coefficient d'aberration sphérique (référé à l'espace objet) et $\alpha$ le demi-angle d'ouverture du faisceau déterminé par un diaphragme fictif situé dans le plan limite des régions I et II. Du fait de la convergence de la région II, le demi-angle d'ouverture $\alpha_{0}$ déterminé par le diaphragme de la première anode est :

$$
\alpha_{0} \# \frac{7}{10} \alpha
$$

- le rayon du disque d'aberration chromatique est (12)

$$
r_{\mathrm{c}}=m C_{\mathrm{c}} \alpha \frac{\Delta V}{V}
$$

$C_{\mathrm{c}}$ étant le coefficient d'aberration chromatique (référé à l'espace objet) et $V$ le potentiel de l'objet $\left(C_{\mathrm{s}}, C_{\mathrm{c}}\right.$ et $V$ sont définis (12) compte tenu du fait que nous avons négligé les effets du champ dans le voisinage immédiat de la pointe de la cathode). Pour $\Delta V$ nous considérons seulement la dispersion totale en énergie des électrons émis par la cathode (13) soit environ $0,2 \mathrm{eV}$, les variations de $V_{0}$ et $V_{i}$ pouvant être rendues négligeables;

- le rayon du disque de diffraction est

$$
r_{\mathrm{d}}=0,61 \frac{\lambda_{\mathrm{i}}}{\alpha_{\mathrm{i}}}
$$

$\lambda_{\mathbf{i}}$ étant la longueur d'onde d'un électron d'énergie e $V_{i}$ et $\alpha_{i}$ le demi-angle d'ouverture dans l'espace image.

Le rayon $\rho$ de la source est alors obtenu en première approximation en faisant la somme quadratique moyenne de toutes ces aberrations.

$$
\rho=\left(r_{\mathrm{g}}^{2}+r_{\mathrm{s}}^{2}+r_{\mathrm{c}}^{2}+r_{\mathrm{d}}^{2}\right)^{1 / 2} .
$$

Pour séparer l'influence du grandissement $\mathrm{m}$ de l'influence des autres paramètres, on définit un rayon de source apparent $\rho_{\mathrm{a}}$

$$
\rho_{\mathrm{a}}=\frac{1}{m}\left(r_{\mathrm{g}}^{2}+r_{\mathrm{s}}^{2}+r_{\mathrm{c}}^{2}+r_{\mathrm{d}}^{2}\right)^{1 / 2}
$$

$\rho_{\mathrm{a}}=\left(r^{2}+C_{\mathrm{s}}^{2} \alpha^{6}+C_{\mathrm{c}}^{2}\left(\frac{\Delta V}{V}\right)^{2} \alpha^{2}+(0,61)^{2} \frac{\lambda^{2}}{\alpha^{2}}\right)^{1 / 2}$ $\lambda$ étant la longueur d'onde d'un électron ayant l'énergie e $\left(R E_{R}\right)$ à la limite des régions I et II. Si $J_{0}$ est la densité de courant électronique à l'émission, l'intensité I du faisceau électronique émise dans l'angle $\alpha$ est :

$$
I=J_{0} \pi R^{2} \alpha^{2}
$$

en supposant que la pointe de la cathode est sphérique de rayon $R$ et que l'émission électronique est répartie uniformément dans l'espace. La densité de courant $J$ dans la source de rayon $\rho$ est :

$$
J=\frac{I}{\pi \rho^{2}}=J_{0} \frac{R^{2} \alpha^{2}}{\rho^{2}} .
$$

La densité de courant par unité d'angle solide ou brillance $\beta$ est alors :

$$
\beta=\frac{J}{\pi \alpha_{\mathrm{i}}^{2}}=\frac{J_{0} R^{2} \alpha^{2}}{\pi \rho^{2} \alpha_{\mathrm{i}}^{2}}=\frac{J_{0} R^{2}}{\pi \rho_{\mathrm{a}}^{2}}\left(\frac{V_{\mathrm{i}}}{R E_{R}}\right)
$$

car :

$$
m=\frac{\alpha}{\alpha_{\mathbf{i}}}\left(\frac{R E_{R}}{V_{\mathrm{i}}}\right)^{1 / 2} .
$$

Cette brillance $\beta$ est donc maximum quand le rayon apparent de la source $\rho_{\mathrm{a}}$ est minimum, les autres paramètres restant fixes bien entendu.

4. Résultats. - Nous avons fait notre étude pour différentes formes d'anodes semblables au modèle de Butler [3], pour deux tensions d'accélérations propres à la microscopie électronique par transmission: $100 \mathrm{kV}$ et $75 \mathrm{kV}$. Les différentes formes d'anodes étudiées sont présentées sur la figure 2 .

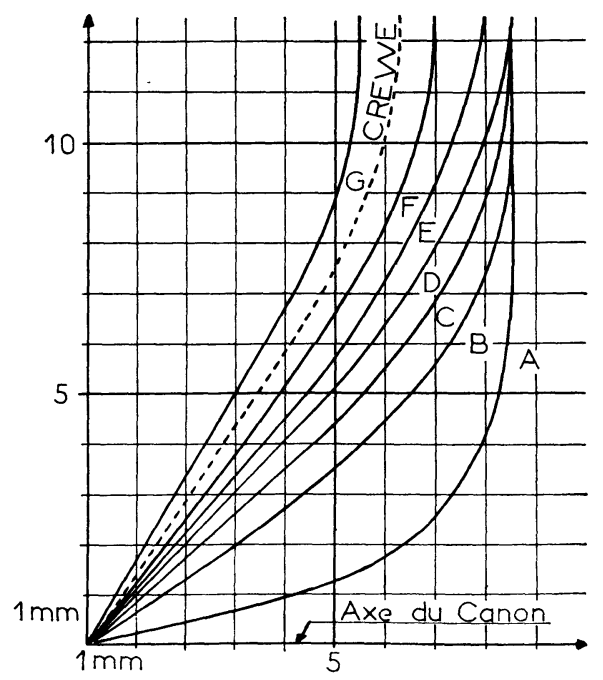

Fig. 2. - Représentation des différentes formes d'anodes étudiées au réseau de résistances.

La distance entre cathode et première anode est $1 \mathrm{~cm}$ et la distance interanodique est $2,2 \mathrm{~cm}$ à $100 \mathrm{kV}$ et $1 \mathrm{~cm}$ à $75 \mathrm{kV}$. Les valeurs choisies pour celle-ci correspondent au seuil des microdécharges dans le vide (14). Nous donnons aussi des résultats concernant deux 
anodes planes, et l'influence de la répartition de potentiel théorique de Becker [10] dans la région II (Fig. 1), ces résultats sont présentés dans les tableaux III et IV.

\section{TABLEAU III}

Les propriétés optiques, les coefficients d'aberration sphérique et chromatique, la brillance $\beta$ optimum sont donnés pour différentes formes d'anodes. La tension d'accélération $V_{\mathrm{i}}=100 \mathrm{kV}$ et le rayon de la pointe $R=0,2 \mu \mathrm{m}$. La dernière colonne correspond à l'anode $\mathrm{D}$, la répartition de potentiel dans la région II du canon étant celle de Becker.

\begin{tabular}{|c|c|c|c|c|c|c|c|c|c|c|}
\hline$\frac{v_{i}}{v_{0}}$ & & A & B & C & D & $E$ & $\mathrm{~F}$ & $G$ & $\begin{array}{l}\text { Anodes } \\
\text { planes }\end{array}$ & Becker \\
\hline 25 & \multirow{3}{*}{$\begin{array}{l}c_{c} \\
(c m)\end{array}$} & $2.4,5$ & 7,43 & 5,53 & 4,68 & 4,7 & 4,8 & 5,8 & 58,5 & 3,8 \\
\hline 18,2 & & & 5,87 & 4,41 & 3,78 & 3,66 & & 3,96 & & \\
\hline 15,3 & & & 5,13 & 3,9 & 3,48 & 3,2 & & 3,26 & & \\
\hline 25 & \multirow{3}{*}{$\begin{array}{l}c_{s} \\
(\mathrm{~cm})\end{array}$} & 0,61 & 0,42 & 0,37 & 0,35 & 0,34 & 0,33 & 0,32 & 0,45 & 0,31 \\
\hline 18,2 & & & 0,39 & 0,35 & 0,33 & 0,32 & & 0,29 & & \\
\hline 15,3 & & & 0,37 & 0,34 & 0,32 & 0,31 & & 0,28 & & \\
\hline \multirow{3}{*}{\begin{tabular}{r|}
25 \\
18,2 \\
15,3 \\
\end{tabular}} & \multirow{3}{*}{$\begin{array}{r}\text { xopt } \\
\left(\begin{array}{c}-3 \\
\left(10^{-3}\right. \\
r d\end{array}\right)\end{array}$} & 3,34 & 4,5 & 4,85 & 5,05 & 5,05 & 5,02 & 4,79 & 2,64 & 5,3 \\
\hline & & & 4,5 & 4,85 & 5,06 & 5,09 & & 4,98 & & \\
\hline & & & 4,61 & 494 & 5,08 & 5,19 & & 5,17 & & \\
\hline 25 & \multirow{3}{*}{$\begin{array}{l}\beta / \mathrm{Jo} \\
\left(x 10^{4}\right. \\
\text { strid }\end{array}$} & 0,92 & 1,47 & 1,67 & 1,79 & 1,82 & 1,84 & 1,79 & 0,7 & 1,96 \\
\hline 18,2 & & & 1,8 & 2 & 2,20 & 2,24 & & 2,26 & & \\
\hline 15,3 & & & 1,8 & 2 & 2,16 & 2,26 & & 2,32 & & \\
\hline 25 & \multirow{3}{*}{$m$} & 0,05 & 0,14 & 0,23 & 0,33 & 0,40 & 0,44 & 0,54 & 0,094 & 0,45 \\
\hline 18,2 & & & 0,7 & 2,65 & 17,4 & $-38,7$ & & $-13,3$ & & \\
\hline 15,3 & & & 11 & -24 & $-8,5$ & $-3,3$ & & $-2,7$ & & \\
\hline 25 & \multirow{3}{*}{$\begin{array}{c}P \\
(\dot{A})\end{array}$} & 7 & 16 & 25 & 35 & 41 & 46 & 56 & 15 & 45 \\
\hline 18,2 & & & 62 & 220 & 1400 & 7600 & & 1050 & & \\
\hline 15,3 & & & 900 & 1800 & 630 & 240 & & 190 & & \\
\hline 25 & \multirow{3}{*}{$\begin{array}{c}d \\
(\mathrm{~cm})\end{array}$} & 0,2 & 1,8 & 3 & 4 & 4,2 & 4,2 & 3,6 & $-0,6$ & 3,7 \\
\hline 18,2 & & & 7,3 & 11,6 & 3,7 & 81,4 & & 61,3 & & \\
\hline 15,3 & & & 10,7 & 194 & 53 & 5,9 & & 2,1 & & \\
\hline
\end{tabular}

On peut représenter l'influence des différentes aberrations en fonction du demi-angle d'ouverture $\alpha$ (Fig. 5). On constate qu'il existe un $\alpha$ optimum pour lequel le rayon apparent de la source $\rho_{\mathrm{a}}$ est minimum donc pour lequel la brillance est maximum. Pour les angles $\alpha$ petits c'est l'aberration de diffraction qui prédomine, puis lorsque $\alpha$ augmente l'aberration chromatique et l'aberration sphérique prennent de l'importance, la dernière étant finalement prépondérante pour les $\alpha$ grands.

Nous avons constaté que c'était surtout la région voisine de l'ouverture anodique de la première anode qui contribuait à l'aberration sphérique. On a donc représenté la variation des coefficients d'aberration sphérique et chromatique, et de la brillance $\beta$ en fonction de la pente de la tangente à la courbe en $\mathrm{O}$ (Fig. 6).

\section{TABLEAU IV}

Les propriétés optiques, les coefficients d'aberration sphérique et chromatique $C_{\mathrm{s}}$ et $C_{\mathrm{c}}$, la brillance $\beta$ optimum sont donnés pour différentes formes d'anodes. La tension d'accélération $V_{\mathrm{i}}=75 \mathrm{kV}$ et le rayon de la pointe $R=0,15 \mu \mathrm{m}$.

\begin{tabular}{|c|c|c|c|c|c|}
\hline$\frac{v_{1}}{v_{0}}$ & $\begin{array}{l}\text { ormes } \\
\text { anodes }\end{array}$ & B & c & D & G \\
\hline \multirow{2}{*}{\begin{tabular}{|l|}
25 \\
18,75
\end{tabular}} & \multirow{3}{*}{$c_{s}$} & 11 & 8,1 & 6,8 & 13 \\
\hline & & 9,1 & 6,6 & 5,5 & 8,8 \\
\hline 15 & & 7,8 & 5,7 & 4,8 & 6,5 \\
\hline 25 & \multirow{3}{*}{$\begin{array}{l}C_{c} \\
(\mathrm{~cm})\end{array}$} & 0,52 & 0,46 & 0,42 & 0,42 \\
\hline \multirow{2}{*}{\begin{tabular}{|c|}
18,75 \\
15 \\
\end{tabular}} & & 0,49 & 0,43 & 0,40 & 0,38 \\
\hline & & 0,46 & 0,41 & 0,38 & 0,35 \\
\hline 25 & \multirow{3}{*}{$\begin{array}{l}\alpha \\
\circ p ! \\
\left(x 10^{-3}\right. \\
\text { rd })\end{array}$} & 4 & 4,4 & 4,6 & 3,9 \\
\hline \multirow{2}{*}{$\frac{18,75}{15}$} & & 4,1 & 4,4 & 4,6 & 4,1 \\
\hline & & 4,1 & 4,5 & 4,7 & 4,3 \\
\hline 25 & \multirow{3}{*}{$\begin{array}{l}\beta / j 0 \\
\left(\times 10^{3}\right. \\
\left.\sin ^{-1} 1\right)\end{array}$} & 5,4 & 6,2 & 6,7 & 5,7 \\
\hline 18,75 & & 6,6 & 7,4 & 8 & 7,2 \\
\hline 15 & & 6,8 & 7,8 & 8,4 & 7,9 \\
\hline
\end{tabular}

\begin{tabular}{|c|c|c|c|c|c|}
\hline for & $m$ & B & C & D & $G$ \\
\hline 25 & \multirow{3}{*}{$5 \mathrm{~m}$} & 0,05 & 0,08 & 0,1 & 0,1 \\
\hline 18,75 & & 0,1 & 0,17 & 0,24 & 0,26 \\
\hline 15 & & 0,22 & 0,48 & 0,85 & 1 \\
\hline 25 & \multirow{3}{*}{$\rho$} & 7 & 9 & 1.1 & 13 \\
\hline 3,75 & & 10 & 16 & 22 & 25 \\
\hline 15 & & 20 & 40 & 70 & 83 \\
\hline 25 & \multirow{3}{*}{ d } & 0,2 & 0,6 & 0,8 & 0,5 \\
\hline 18,75 & & 1,2 & 2 & 2,6 & 2 \\
\hline 15 & & 2,8 & 4,5 & 6 & 4,6 \\
\hline
\end{tabular}

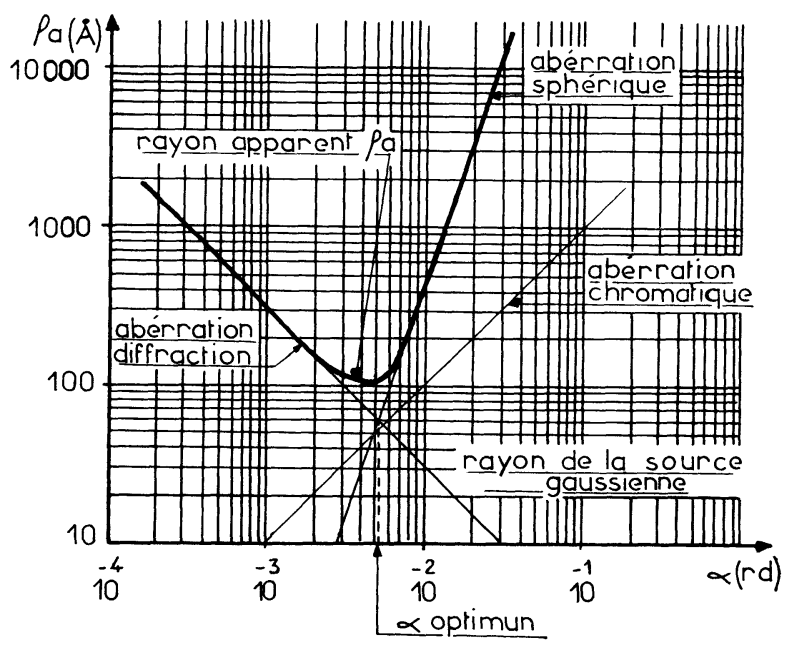

FIG. 5. - Représentation des différentes limites aürayon $\rho_{\mathrm{a}}$ de source apparente. Variation de $\rho_{\mathrm{a}}$ en fonction du demi"angle d'ouverture.

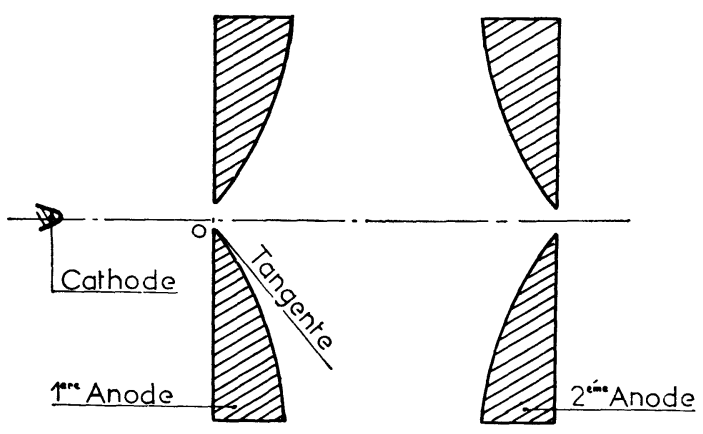

FIG. 6. - Représentation de la tangente à la première anode en $O$. La pente $p$ de cette tangente est le paramètre porté en abscisse dans la figure 7 . 
4.1 RÉSULTATS POUR LA TENSION D'ACCÉLÉRATION $V_{\mathrm{i}}=100 \mathrm{kV}$. - Le coefficient d'aberration sphérique passe par un minimum (Fig. 7). Ce minimum n'est pas le même suivant le rapport de tensions $V_{\mathrm{i}} / V_{0}$ : il se décale de la forme d'anode $\mathrm{D}$ à la forme $\mathrm{F}$ aqund $V_{\mathrm{i}} / V_{0}$ passe de 25 à 15,3 (Fig. 8). La valeur du coefficient d'aberration sphérique pour deux anodes planes séparées d'une distance de $2,2 \mathrm{~cm}$ est $58,5 \mathrm{~cm}$, soit 12 fois plus grande que celle du coefficient d'aberration sphérique minimum, il y avait donc intérêt à rechercher les conditions d'extrémum.

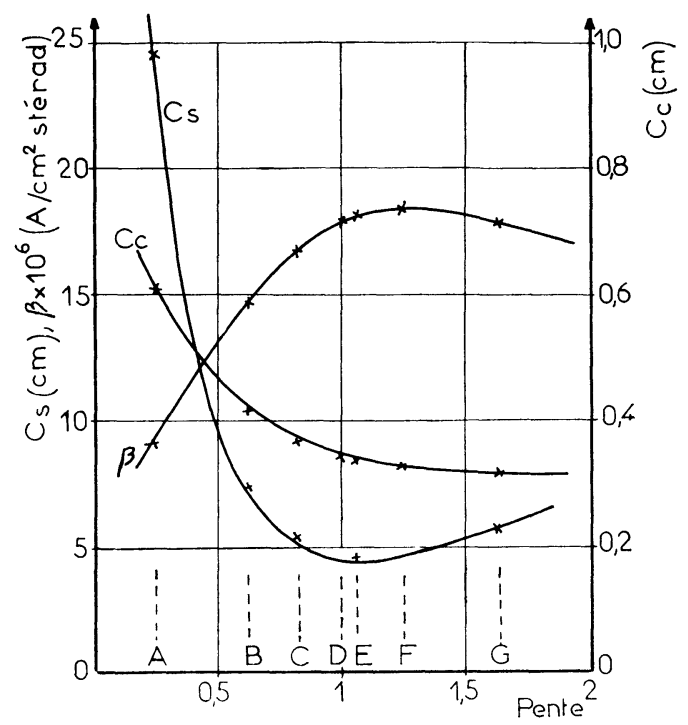

Fig. 7. - Variation des coefficients d'aberration sphérique et chromatique $C_{\mathrm{s}}$ et $C_{\mathrm{c}}$ et de la brillance $\beta$ en fonction de la pente $p$ de la tangente aux anodes en 0 (Fig. 6). $V_{\mathrm{i}} / V_{0}=25.7$

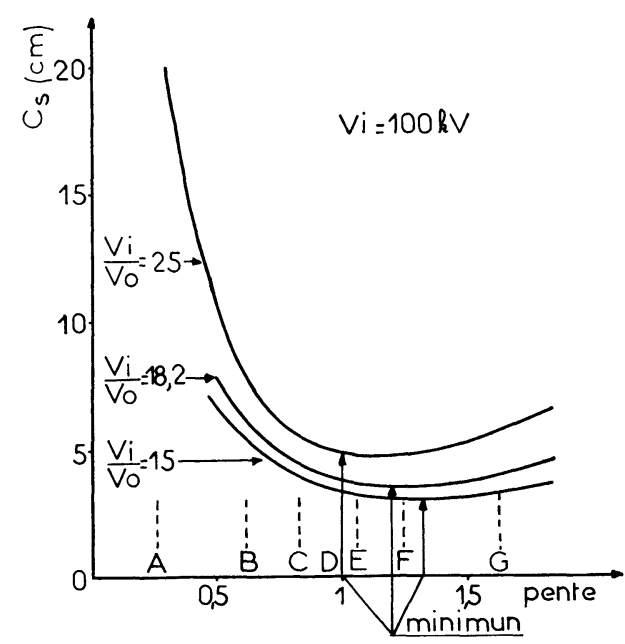

Fig. 8. - Variation de la position du minimum de $C_{\mathrm{s}}$ pour différents rapports $V_{\mathbf{i}} / V_{0}$.

Le coefficient d'aberration chromatique décroît constamment quand la pente de la tangente aux anodes en $\mathrm{O}$ augmente (Fig. 7), mais cette décroissance est très peu sensible de la forme $\mathrm{C}$ à la forme $\mathrm{G}$. En fait la valeur de $C_{\mathrm{c}}$ dépend beaucoup moins de la forme d'anode que le coefficient d'aberration sphérique : en effet, pour des anodes planes il est égal à $0,45 \mathrm{~cm}$ soit seulement $1 / 4$ de fois plus grand que le plus petit.

Il est à noter que la brillance passe par un maximum qui ne correspond pas au minimum du coefficient d'aberration sphérique car les autres aberrations ont une influence non négligeable. Ce maximum se déplace de la forme $\mathrm{F}$ à la forme $\mathrm{G}$ quand le rapport de tensions $V_{\mathrm{i}} / V_{0}$ diminue. Dans les tableaux III et IV nous avons représenté $\beta / J_{0}, J_{0}$ étant la densité de courant à l'émission. Lorsque le rapport $V_{\mathrm{i}} / V_{0}$ diminue de 25 à $15,3, J_{0}$ varie de $10^{3} \mathrm{~A} / \mathrm{cm}^{2}$ à $10^{6} \mathrm{~A} / \mathrm{cm}^{2}$. La brillance augmente donc de $10^{7} \mathrm{~A} / \mathrm{cm}^{2}$ stérad. à $10^{10}$ $\mathrm{A} / \mathrm{cm}^{2}$ stérad.

En pratique la densité de courant $J_{0}$ à laquelle on travaillera est $10^{5} \mathrm{~A} / \mathrm{cm}^{2}$ (pour des valeurs plus grandes la stabilité relative du courant d'émission peut être fortement perturbée (15) à cause d'une remontée de pression dans l'enceinte due au bombardement électronique trop intense des anodes), ce qui donne une brillance de l'ordre de $10^{9} \mathrm{~A} / \mathrm{cm}^{2}$ stérad.

Le grandissement varie énormément suivant le rapport de tensions $V_{\mathrm{i}} / V_{0}$ (Fig. 9). Pour certaines valeurs de ce rapport il peut même devenir très grand et changer de signe lorsque le rapport $V_{\mathrm{i}} / V_{0}$ décroît encore.

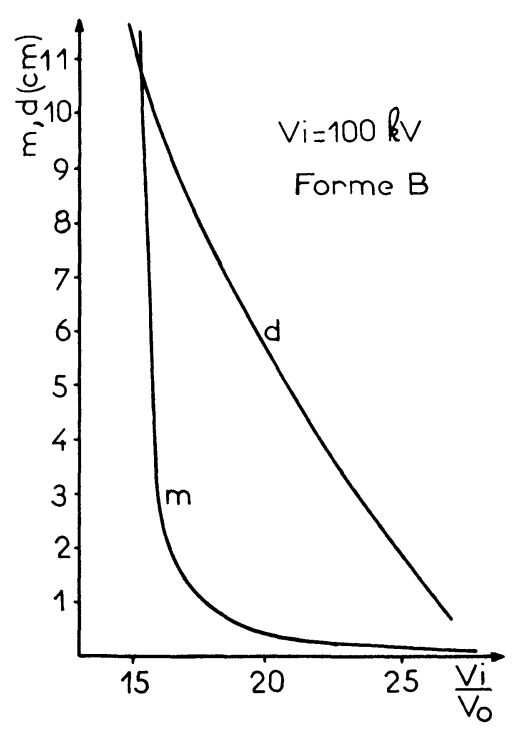

FIG. 9. - Variations du grandissement $m$ et de la position de la source d pour la forme d'anode $\mathrm{B}$ en fonction du rapport $V_{\mathrm{i}} / V_{0}$.

Le rayon de la source dépend essentiellement de la valeur du grandissement. Il peut prendre de très petites valeurs $(\sim 10 \AA)$ pour des rapports de tensions $V_{\mathrm{i}} / V_{0}$ élevés.

La distance $d$ de la source à la deuxième anode (sens positif sur la Fig. 1) augmente quand le rapport $V_{\mathrm{i}} / V_{0}$ diminue pour les formes A et B (Fig. 9). Pour les autres formes la variation n'est pas uniforme, pour une certaine valeur de $V_{\mathrm{i}} / V_{0}$ cette distance devient très grande et tend même vers l'infini. En pratique il faudra 
donc choisir une forme d'anode qui ne présente pas cet inconvénient à la tension $V_{0}$ à laquelle on a choisi de travailler.

4.2 RÉSULTATS POUR LA TENSION D'ACCÉLÉRATION. $V_{\mathrm{i}}=75 \mathrm{kV}$. - Les résultats présentés dans le tableau IV ont été calculés pour un rayon de la pointe $R=0,15 \mu \mathrm{m}$. Le champ électrostatique appliqué à celle-ci est alors le même qu'à $100 \mathrm{kV}$ et les résultats sont donc comparables.

De manière générale les variations des coefficients d'aberration et de la brillance sont les mêmes qu'à $100 \mathrm{kV}$.

Ces coefficients d'aberration sont plus grands à $75 \mathrm{kV}$, alors que la brillance est plus petite.

Le grandissement ne présente pas de changement de signe et reste très petit, si bien que le rayon de la source ne dépasse pas une centaine d'Angströms.

La distance de la source à la deuxième anode reste dans des limites convenables puisque sa valeur maximum est $4,6 \mathrm{~cm}$ pour l'anode $\mathrm{G}$ et un rapport $V_{\mathrm{i}} / V_{0}=15$.

4.3 COMPARAISON AVEC LA FORME D'ANODE THÉORIQUE DE CREWE. - Crewe [7] a donné deux formules qui permettent de préciser l'anode qui procure un coefficient d'aberration sphérique minimum :

- l'une de ces formules fixe la géométrie des anodes $(*)$;

- l'autre donne la répartition du potentiel entre les anodes qui correspond à la forme déterminée par la formule précédente.

Pour comparer notre méthode expérimentale à celle de Crewe, nous avons mesuré point par point la répartition de potentiel entre les anodes correspondant à la forme d'anode donnée par celui-ci, pour une tension

\section{TABleau $\mathbf{X}$}

Valeurs expérimentales et valeurs théoriques du potentiel $V(z)$ sur l'axe pour la forme d'anode de Crewe :

$$
V_{(z=1)}=100000 \mathrm{~V}, \quad V_{(z=0)}=4000 \mathrm{~V} \text {. }
$$

Les valeurs sont données pour $z$ variant de 0 à $1 / 2$.

\begin{tabular}{|c|c|c|c|c|c|c|c|c|c|c|}
\hline$z$ & 0 & 0,054 & 0,108 & 0,162 & 0,216 & 0,270 & 0,324 & 0,378 & 0,432 & 0,500 \\
\hline $\begin{array}{l}\text { Valeurs expé- } \\
\text { rimentales de } \\
V(Z)\end{array}$ & 4000 & 4792 & 7218 & 11026 & 15976 & 21850 & 28396 & 35416 & 42700 & 52000 \\
\hline $\begin{array}{l}\text { Voleurs s rhé } \\
\text { riques de } \\
\vee(Z) \text { (volts) }\end{array}$ & 4000 & 4710 & 7123 & 10749 & 15520 & 21242 & 27750 & 36831 & 42339 & 52000 \\
\hline
\end{tabular}

(*) Il est à noter que la géométrie des anodes dépend de la distance entre les deux diaphragmes anodiques, mais assez faiblement puisque pour une distance entre diaphragmes variant de $2 \mathrm{~cm}$ à $4 \mathrm{~cm}$ (correspondant à des tensions accélératrices variant de $30 \mathrm{kV}$ à $100 \mathrm{kV}$ ) la forme reste inscrite entre les formes $\mathrm{F}$ et $\mathrm{G}$ (Fig. 2). d'accélération de $100 \mathrm{kV}$. Nos résultats comparés à la répartition de potentiel théorique tirée de Crewe sont en bon accord (tableau X), l'écart entre les valeurs théoriques et expérimentales est toujours inférieur à $3 \%$.

Toutefois, il faut remarquer que la méthode employée par Crewe pour déterminer la forme optimum d'anode est assez restrictive. En effet, une fois qu'on s'est fixé la distance entre diaphragmes (c'est-à-dire une certaine tension d'accélération à ne pas dépasser), la forme d'anode est imposée d'une manière unique. Ceci sous-entend donc que le coefficient d'aberration sphérique est minimum quelles que soient les conditions de fonctionnement du canon, c'est-à-dire quel que soit le rapport $V_{\mathrm{i}} / V_{0}$ auquel on travaille.

En fait, nos résultats expérimentaux montrent qu'il n'y a pas une forme et une seule qui donne un coefficient d'aberration sphérique minimum mais que, suivant les valeurs du rapport $V_{\mathrm{i}} / V_{0}$, cette forme peut varier.

5. Discusion. - Suivant l'utilisation qu'on voudra faire d'un canon à émission de champ, un choix de la forme d'anode adéquate pourra être fait grâce aux résultats présentés dans les tableaux III et IV. Ce choix pourtant ne doit pas être essentiellement guidé, comme on pourrait le croire d'après Crewe, pour la recherche uniquement d'un coefficient d'aberration sphérique minimum. En effet, les autres aberrations n'ont pas un rôle complètement négligeable puisque le maximum de brillance est obtenu pour une forme différente de celle qui donne un coefficient d'aberration sphérique minimum. Généralement dans tous les domaines de la microscopie électronique, particulièrement en microscopie à balayage, en microscopie haute résolution et en microscopie interférentielle l'amélioration de la brillance (*) est un facteur prépondérant. C'est donc la brillance qui déterminera le choix des anodes. Le diamètre de la source électronique peut aussi intervenir dans ce choix (surtout en microscopie à balayage) mais notons qu'il est toujours possible de réduire ses dimensions en utilisant des lentilles magnétiques condenseurs. (Grâce à leurs coefficients d'aberration très petits, elles n'affectent presque pas les qualités du faisceau électronique.)

Dans le cas de la microscopie électronique à $75 \mathrm{kV}$, le rapport de tensions $V_{\mathrm{i}} / V_{0}$ généralement utilisé étant voisin de 15 , la forme d'anode choisie pourra être la forme F (Fig. 2).

Pour la microscopie électronique à $100 \mathrm{kV}$, le choix s'avère plus difficile.

Grâce au procédé de formation des pointes décrit par Bettler et Charbonnier [11] la tension $V_{0}$ appliquée

(*) Avec une grande brillance on peut avoir une source électronique de dimension très petite (microscopie à balayage) ou améliorer la cohérence transverse du faisceau électronique (microscopie haute résolution et interférentielle), tout en ayant une densité de courant sur l'objet qui permette de faire une microphotographie avec un temps de pose raisonnable. 
à celles-ci pour avoir une densité de courant à l'émission $J_{0} \# 10^{5} \mathrm{~A} / \mathrm{cm}^{2}$ restera assez faible permettant de travailler à des rapports de tensions $V_{\mathrm{i}} / V_{0}$ supérieurs à 20. Pour de tels rapports de tensions la forme $F$ donne le maximum de brillance, mais présente des inconvénients quant à la taille du cross-over et à la position de celui-ci. En effet pour le rapport $V_{\mathrm{i}} / V_{0}=18,2$, le grandissement change de signe entre les formes $\mathrm{D}$ et $E$ et devient grand, le rayon du cross-over dépasse plusieurs milliers d'Angströms et sa position plusieurs dizaines de centimètres. La forme $\mathrm{D}$ ne présente pas ces invonvénients pour des rapports $V_{\mathrm{i}} / V_{0}$ supérieurs à 20 et la brillance qu'elle permet d'obtenir est très peu inférieure à celle que donne la forme F. Nous avons vu (Fig. 5) que l'aberration sphérique était prédominante pour des demi-angles d'ouverture $\alpha$ grands (En effet $\alpha$ intervient au cube dans l'aberration sphérique.)

Or, plus l'angle $\alpha$ est petit (c'est-à-dire plus le diamètre du diaphragme d'anode est petit), plus le centrage du faisceau électronique sur l'axe du microscope est difficile. On se rend donc compte que le choix d'un diaphragme d'anode de diamètre supérieur à celui du diaphragme optimum cause une perte en brillance très rapide.

La forme D donne un coefficient d'aberration sphérique minimum et un demi-angle d'ouverture maximum ce qui ne saurait être qu'un avantage.

6. Conclusion. - Nous avons étudié l'étage accélérateur d'un canon à émission de champ par la méthode analogique du réseau de résistances. Nous avons recherché qu'elle était la forme d'anode qui permettait l'obtention de la brillance maximum, et constaté que cette forme variait quelque peu suivant les tensions de fonctionnement appliquées aux anodes. Les grandeurs électrooptiques de celles-ci : grandissement linéaire, diamètre et position de la source électronique ont été également déterminés. La brillance maximum permise par de telles anodes à la tension d'accélération de $100 \mathrm{kV}$ et pour une densité de courant à l'émission de $10^{5} \mathrm{~A} / \mathrm{cm}^{2}$ est de l'ordre de $10^{9} \mathrm{~A} / \mathrm{cm}^{2}$ stérad. Ceci constitue un gain d'environ $10^{3}$ par rapport aux canons conventionnels équipés de cathodes à pointe chauffée.

\section{Bibliographie}

[1] Everhart (T. E.), J. Appl. Phys., 1967, 38, 4944.

[2] WORSTER (J.), Int. J. Electronics, 1969, 27, 49.

[3] Butler (T. W.), Sixth Inter. Congr. Electron Microscopy, Kyoto, 1, 193.

[4] Crewe (A. V.), EgGenberger (D. N.), Wall (J.) et Welter (L. M.), The Rev. of Scient. Inst., 1967, $39,576$.

[5] Wiesner (J. C.) et Everhart (T. E.), 7e Cong. Inter. Microscopie électronique, Grenoble, 1970, 85.

[6] Dyke (W. P.), Trolan (J. K.), Dolan (W. W.) et Barnes (G.), J. Appl. Phys., 1953, 24, 570.

[7] Crewe (A. V.), Quarterly Reviews of Biophys., 1970, 3, 137

[8] Troyon (M.), Bonhomme (P.) et Bonnet N.), colloque de Caen, J. de Microscopie, 1971, 11, 20.
[9] Troyon (M.), thèse $3^{\mathrm{e}}$ cycle, Faculté des Sciences Reims, juillet 1971.

[10] Becker (J. A.), The Bell. Syst. Technical J. 907-932, 1951.

[11] Bettler (P. C.) et Charbonnier (F. M.), Phys. Rev., $1960,119,85$.

[12] Grivet (P.), Optique électronique I (ed. Bordas), 1955,80

[13] Young (R. D.) et Muller (F. W.), Phys. Rev., 1959, 113, 115.

[14] Arnal (R.), Annales de Phys., 12 séries, 1955, 10, 830.

[15] Swanson (L. W.) et Crouser (L. C.), J. of. Appl. Phys., 1969, 40, 4741. 